\title{
Novel flower-shaped albumin particles as controlled-release carriers for drugs to penetrate the round-window membrane
}

This article was published in the following Dove Press journal:

International Journal of Nanomedicine

I July 2014

Number of times this article has been viewed

\section{Zhan Yu ${ }^{1, *}$ \\ Min $\mathrm{Yu}^{2, *}$ \\ Zhimin Zhou ${ }^{3}$ \\ Zhibao Zhang ${ }^{3}$ \\ Bo $\mathrm{Du}^{3}$ \\ Qingqing Xiong ${ }^{3}$}

'Second Artillery General Hospital, Beijing, ${ }^{2}$ Department of Cell Biology, Key Laboratory of Cell Biology, Ministry of Public Health, College of Basic Medicine, China Medical University, Shenyang, ${ }^{3}$ Institute of Biomedical Engineering, Chinese Academy of Medical Sciences, Peking Union Medical College, Key Laboratory of Biomedical Material of Tianjin, Tianjin, People's Republic of China

*These authors contributed equally to this work
Correspondence: Zhan Yu Second Artillery General Hospital, 16 Xinjiekou Outer Avenue, Beijing 100088, People's Republic of China $\mathrm{Tel}+86$ I0 242I 3139

Fax +86 I0 242I 3139

Email yuzhandz@I26.com

Min Yu

China Medical University, 92 Beier Road, Shenyang, I I000 I, People's Republic of China

Tel +86242326I056

Fax +86242326 1056

Emailym77@I63.com

\begin{abstract}
Controlled-release carriers for local drug delivery have attracted increasing attention for inner-ear treatment recently. In this paper, flower-shaped bovine serum albumin (FBSA) particles were prepared by a modified desolvation method followed by glutaraldehyde or heat denaturation. The size of the FBSA particles varied from $10 \mu \mathrm{m}$ to $100 \mu \mathrm{m}$, and most were $50-80 \mu \mathrm{m}$. Heat-denatured FBSA particles have good cytocompatibility with a prolonged survival time for L929 cells. The FBSA particles were utilized as carriers to investigate the release behaviors of the model drug - rhodamine B. Rhodamine B showed a sustained-release effect and penetrated the round-window membrane of guinea pigs. We also confirmed the attachment of FBSA particles onto the round-window membrane by microscopy. The FBSA particles, with good biocompatibility, drug-loading capacity, adhesive capability, and biodegradability, may have potential applications in the field of local drug delivery for inner-ear disease treatment.
\end{abstract}

Keywords: bovine serum albumin (BSA), controlled release, local delivery, round-window membrane

\section{Introduction}

Inner-ear disorders, including sensorineural hearing loss, occur commonly in the clinical setting. Traditional systemic therapies are almost ineffective, due to the blood-labyrinth barrier. The round-window membrane (RWM) is a semipermeable membrane located between the middle-ear cavity and the inner-ear perilymphatic fluid. ${ }^{1}$ It allows drugs less than $3 \mu \mathrm{m}$ in diameter to penetrate it. Therefore, local drug delivery, especially via intratympanic (IT) injection, has become popular due to its efficiency and safety. ${ }^{1}$

Local drug delivery to the inner ear by IT injection was first described by Schuknecht in 1956 in the treatment of Ménière's disease. ${ }^{2}$ In 2001, Kopke et al reported a significant hearing improvement in patients with sudden sensorineural hearing loss after microcatheter administration of methylprednisolone. ${ }^{3}$

Although IT injection is easy to perform in the clinic, the loss of drug through the eustachian tube suppresses the efficiency of treatment for inner-ear disorders. Therefore, drug-delivery vehicles have been investigated for a sustained and prolonged supply. In 1998, Balough et al reported that fibrin-based vehicles impregnated with gentamicin were successfully injected into the middle ear of chinchillas, and allowed for a prolonged effect without absorption in the untreated ear or blood. ${ }^{4}$ In 2010, Horie et al reported that drug-loaded poly(lactic-co-glycolic acid) (PLGA) microparticles were capable of delivering lidocaine into the cochlea in a sustained manner. ${ }^{5}$ The PLGA nanoparticles (NPs) were found to be 
distributed throughout the inner ear after injection in the RWM of chinchilla. ${ }^{6}$ Moreover, Tan et al demonstrated that brain-derived neurotrophic factor encapsulated in poly(Lglutamic acid) particles could be released in a sustained manner with maintained biological activity. ${ }^{7}$ The brainderived neurotrophic factor released efficiently rescued primary auditory neurons of guinea pigs with sensorineural hearing loss.

Recently, there has been growing recognition that the shape of drug carriers plays an essential role in the design of materials with certain properties. Nonspherical particles have attracted increasing attention for their potential applications in drug-delivery systems. ${ }^{8}$ Many methods, such as film hydration, emulsion, template, microfluidic, mechanical stretching, and self-assembly can be used in the preparation of nonspherical particles. ${ }^{9-18}$ It is of great importance to develop a simple method to prepare nonspherical particles with a high yield. ${ }^{8}$ In this study, we demonstrated that bovine serum albumin (BSA) can form flower-shaped microparticles via modified desolvation fabricated with template and salting-out methods.

BSA is a natural protein able to form complexes with several molecules. ${ }^{19}$ This protein is biocompatible, biodegradable, and nonimmunogenic. Due to these features, albumin particles are convenient systems for drug delivery. ${ }^{12,19-21} \mathrm{Ge}$ et al reported a method for creating hybrid organic-inorganic nanoflowers using copper(II) ions and proteins including BSA. ${ }^{6}$ However, so far there have been no reports of flower-shaped particles made of BSA with a high yield and a simple procedure. There have been no reports either of such microparticles for local drug delivery to the inner ear. Here, we illustrate a method for creating flower-shaped BSA (FBSA) microparticles with biocompatible and adhesive capability with a high yield. A model drug (rhodamine B [RhB]) was loaded onto the FBSA for drug-loading and release studies. In vivo biodistribution suggested that RhB released from FBSA tended to accumulate for a long time on the RWM of guinea pigs. Therefore, FBSA particles are promising controlled-release carriers for local drug delivery in the treatment of inner-ear disorders.

\section{Materials and methods}

\section{Materials, animals, and cell culture}

BSA, RhB, doxorubicin, and fluorescein isothiocyanate (FITC)-BSA were purchased from Sigma-Aldrich (St Louis, MO, USA). A Cell Counting Kit (CCK)-8 was purchased from Dojindo Molecular Technologies (Mashiki,
Japan), and prednisolone was purchased from Tianjin Pharmaceuticals (Tianjin, People's Republic of China [PRC]). Ultrapure water used in all experiments was produced by Milli-Q ${ }^{\circledR}$ synthesis system (EMD Millipore, Billerica, MA, USA).

Guinea pigs weighting 250-300 g were purchased from the Tianjin Experimental Animal Center, Tianjin, PRC, and had free access to food and water. Animal-study protocols were approved and performed in accordance with the recommendations in the Guide for the Care and Use of Laboratory Animals. ${ }^{22}$ L929 mouse fibroblast cells (obtained from the Cancer Institute of the Chinese Academy of Medical Sciences, Beijing, PRC) were cultured in Dulbecco's Modified Eagle's Medium (HyClone ${ }^{\text {TM}}$; Thermo Fisher Scientific, Waltham, MA, USA) containing 10\% fetal bovine serum at $37^{\circ} \mathrm{C}$ and $5 \% \mathrm{CO}_{2}$.

\section{Preparation of FBSA- and RhB-loaded FBSA}

A modified desolvation method was used to prepare the FBSA microparticles. BSA (100 mg) was dissolved in $1 \mathrm{~mL}$ of carbonate solution (0.1 M, pH 8.4). Then, $8.0 \mathrm{~mL}$ of ethanol was added dropwise (within 1 minute) into the mixture under magnetic stirring $(400 \mathrm{rpm})$ at room temperature. Subsequently, the as-prepared FBSA particles were crosslinked with $0.2 \%$ glutaraldehyde (GA) for 24 hours or denatured at $70^{\circ} \mathrm{C}$ for 6 hours by continuous stirring. The FBSA particles were centrifuged and washed with ultrapure water. FBSA $(50 \mathrm{mg}$ ) was incubated with certain amounts $(5,10,15,17.5$, and $20 \mathrm{mg})$ of RhB for 2 hours in the preparation of RhB-loaded FBSA (FBSA-RhB).

\section{Characterization of the FBSA particles}

Morphological characteristics were determined by transmission electron microscopy (TEM; JEM-100CX II; JEOL, Tokyo, Japan), scanning electron microscopy (SEM; Supra 55VP; Carl Zeiss Meditec, Jena, Germany) and confocal laser-scanning microscopy (CLSM; FV-1000; Olympus, Tokyo, Japan). For TEM, a drop of diluted suspension of FBSA was placed on a copper grid, and the air-dried specimen was observed. For SEM, a drop of diluted suspension was deposited on a silicon wafer. The air-dried sample was coated with gold and observed. FBSA-RhB particles were observed by CLSM at an excitation wavelength of $555 \mathrm{~nm}$ and an emission wavelength of $580 \mathrm{~nm}$.

The FBSA particles were dispersed in ultrapure water at a concentration of $0.1 \mathrm{mg} / \mathrm{mL}$. Zeta potential was determined by a Zetasizer Nano ZS particle-size analyzer (Malvern 
Instruments, Malvern, UK). FBSA particles with or without desalting were air-dried and observed by SEM. An energy-spectrum analysis was performed on the "petals" to qualify and quantify the elemental composition.

FBSA particles fixed with GA were divided into three parts: one for control, one for desalting, and another one for reaction with $\mathrm{CaCl}_{2}$. The three specimens were dried by evaporation, and confirmed by an X'Pert Pro X-ray diffractometer (Spectris, Egham, UK).

\section{Drug-loading capacity and encapsulation efficiency}

FBSA particles $(50 \mathrm{mg})$ with heat denaturation were incubated with $\mathrm{RhB}$ (5-20 mg) for 2 hours. After being washed with ultrapure water, the supernatants were collected and analyzed for residual drug concentration by ultraviolet-visible analysis.

Drug-loading capacity and encapsulation efficiency were calculated as follows:

drug-loading $(\mathrm{w} / \mathrm{w} \%)=$ amount of $\mathrm{RhB}$ in FBSA/amount of FBSA $\times 100$;

encapsulation efficiency $(\mathrm{w} / \mathrm{w} \%)=$ amount of $\mathrm{RhB}$ in FBSA/RhB initially added $\times 100$.

\section{In vitro drug-release behavior}

$\mathrm{RhB}$ release was evaluated in a standard static diffusion cell at a speed of $100 \mathrm{rpm}$ in a shaker at $37^{\circ} \mathrm{C}$. The amount of $\mathrm{RhB}$ was evaluated using an ultraviolet-visible spectrometer $(560 \mathrm{~nm})$. The amount of $\mathrm{RhB}$ released was evaluated at a series of time points ( $0-120$ hours), and then the release curve was made accordingly.

\section{Cell-biocompatibility assay}

Cells were seeded in 96-well plates at a density of 1,000 cells/well, and further cultured for 24 hours. FBSA-GA or heat-denatured FBSA particles (FBSA-H) were added to each well for a 24-hour incubation. Cell viability was determined by CCK- 8 and quantified by the ratio to the control. Untreated cells served as $100 \%$ cell viability. The morphology of L929 cells in each group was also observed by phase-contrast microscopy. After that, cells were cultured continuously for at least 7 days without changing the culture medium and observed by microscope.

\section{In vivo assay}

Guinea pigs were killed to sample the acoustic bullae (including the RWM). The acoustic bullae were placed in solution of
FBSA and BSA NPs (the preparation of which was described in another paper by the same authors being reviewed) and shaken for 30 minutes at $37^{\circ} \mathrm{C}$. The air-dried specimens were observed by SEM.

The penetration of $\mathrm{RhB}$ released from the particles was evaluated by living images and microscopes. The same amount of RhB was incubated with FBSA-H particles and BSA NPs in the preparation of FBSA-RhB and BSA-NP-RhB. Guinea pigs were anesthetized, and the RWMs were exposed. The FBSA-RhB and BSA-NP-RhB particles dispersed in phosphate-buffered saline were injected slowly into the bulla of the right and left ear, respectively. The left ear injected with BSA-NP-RhB was the control. An in vivo imaging system (Caliper IVIS ${ }^{\circledR}$; PerkinElmer, Waltham, MA, USA) was used to trace the particles at time points of 0 and 48 hours. RWMs were then imaged by fluorescence microscopy to observe the distribution of RhB on them. Several days later, RWMs with particles were observed by SEM.

\section{Statistical analysis}

The statistical data obtained are presented as mean values and standard deviation. Student's $t$-test was used in SPSS 12.0 (SPSS, Chicago, IL, USA) to determine significant differences between groups, and $P$-values less than 0.05 were considered statistically significant.

\section{Results and discussion Morphology of FBSA particles}

During the preparation of BSA NPs by desolvation, we discovered stable FBSA particles by accident (Figure 1A). Figure 1 shows typical FBSA-GA (Figure 1B) and FBSA-H (Figure 1C) particles. Moreover, the corresponding TEM image (Figure 1F) confirms that the particles showed a radical pattern. The size of these particles varied from $10 \mu \mathrm{m}$ to $100 \mu \mathrm{m}$; most of them were 50-80 $\mu \mathrm{m}$. As shown in Figure 1B, FBSA-GA particles were also in the shape of a flower, but changed slightly, maybe due to the stirring. Therefore, we can conclude that the morphology of FBSA particles shows no obvious difference whether treated by heat or GA. However, there was little difference among the particles viewed by the naked eye; the colors of the precipitates were yellow (Figure 1B, inset) and milk white (Figure 1C, inset), respectively.

RhB was used as a model drug for observation and evaluation of drug-loading capacity. The fluorescent image of FBSA-RhB (Figure 2A) confirms that RhB is adsorbed on the FBSA. The FBSA and FBSA-RhB particles had zeta-potential values of $-16.2 \mathrm{mV}$ and $+0.298 \mathrm{mV}$, respectively. The potential difference dem- 

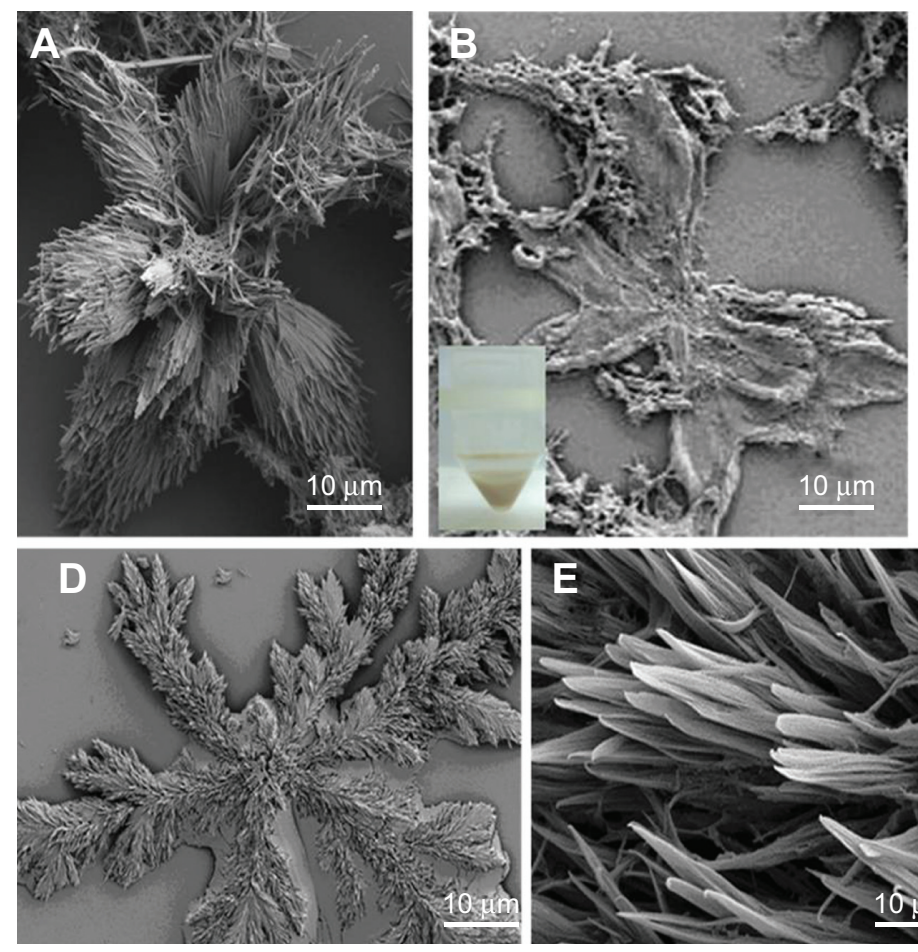

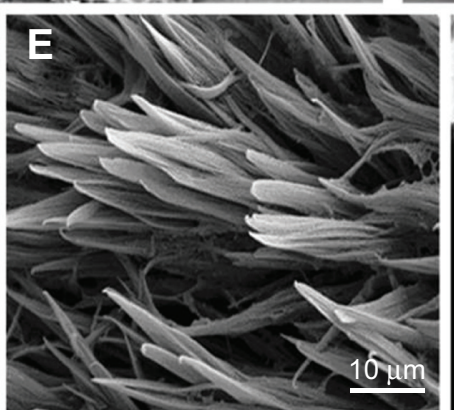

Figure I Structure of FBSA particles by electronic microscope.

Notes: FBSA particles before fixation (A) and after fixation by glutaraldehyde $(\mathbf{B})$ and denatured by heat $(\mathbf{C})$ are demonstrated by SEM. The appearance of FBSA-GA (b inset) and FBSA-H (c inset) are also shown in the insets. The SEM images of $\mathrm{NaHCO}_{3}$ crystal are demonstrated in (D) and (E). The TEM image of FBSA denatured by heat is demonstrated in (F).

Abbreviations: FBSA, flower-shaped bovine serum albumin; SEM, scanning electron microscopy; FBSA-GA, glutaraldehyde-fixed FBSA; FBSA-H, heat-denatured FBSA; TEM, transmission electron microscopy.

onstrated that the positively charged RhB might have had an interaction with the negatively charged BSA, which also promoted the attachment of RhB to the FBSA. We also prepared doxorubicin-loaded FBSA (Figure 2B), which also showed the same adsorption effect. Therefore, the model drug and small molecules affected certain parameters (such as size, shape, charge) of particles, which was in agreement with previous reports. ${ }^{8,23-25}$ Then, we used FITC-BSA to prepare FITC-FBSA particles according to the aforementioned method. The shape of the FITC-FBSA particles (Figure 2C) was almost the same as those of FBSA.

\section{Mechanism of BSA formation in flower shape}

We repeated the aforementioned preparation procedure with $\mathrm{NaHCO}_{3}$ only (hence, without the presence of BSA) and compared the shape with that of FBSA. As shown in Figure 1D, it was also in the shape of a flower very similar to that of the FBSA (Figure 1A-C). We assumed that this was due to the BSA salting out in the presence of ethanol and adhering to the template of flower-shaped $\mathrm{NaHCO}_{3}$ crystals, which was then confirmed by X-ray diffraction analysis. As shown in Figure 1, the shape of BSA was mainly correlated

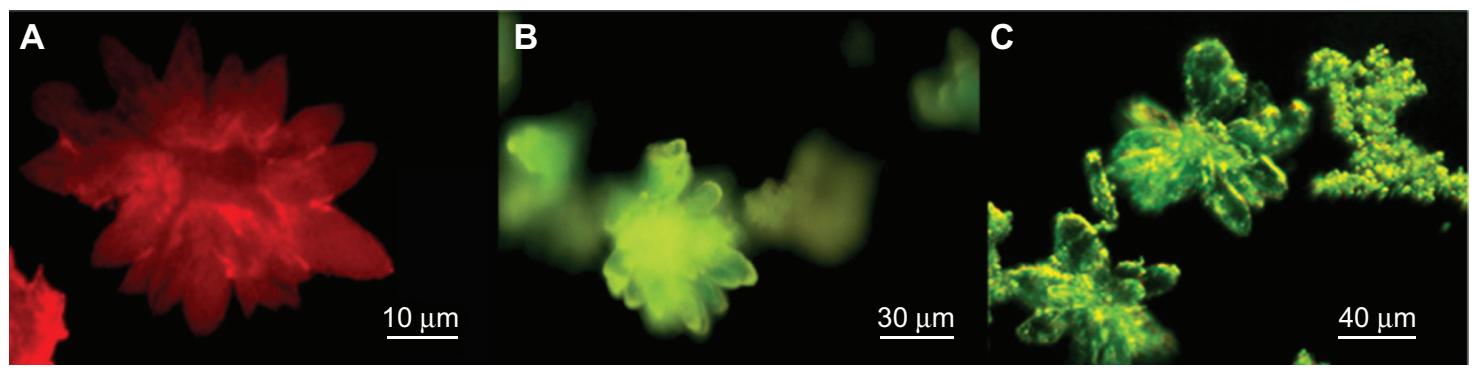

Figure 2 Drug-loaded FBSA observed by microscopy.

Note: The rhodamine B-loaded FBSA (A), doxorubicin-loaded FBSA (B), and fluorescein isothiocyanate-loaded FBSA (C) particles are displayed by fluorescent microscopy. Abbreviation: FBSA, flower-shaped bovine serum albumin. 


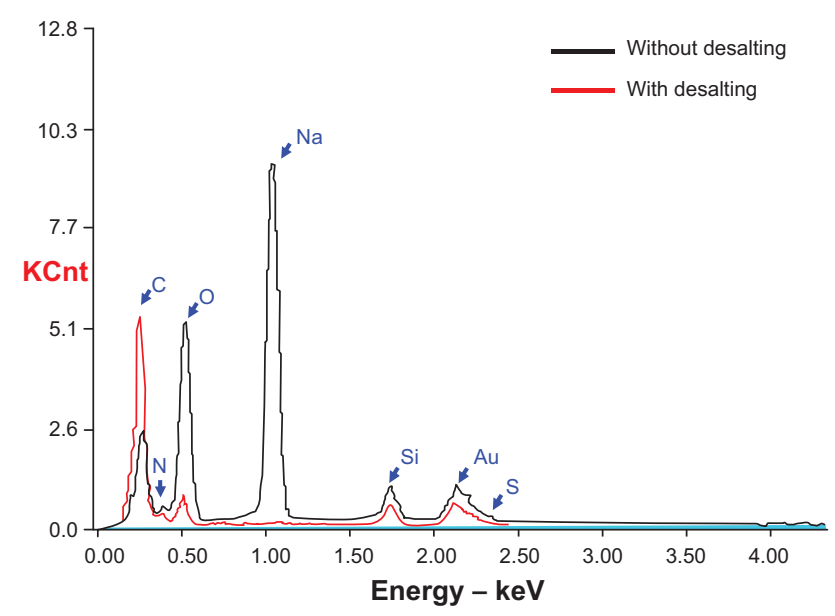

Figure 3 Energy-spectrum analysis of FBSA with or without desalting. The sodium is absent after desalting, while the carbon is elevated obviously after desalting. Carbon, nitrogen, and sulfur are present in both specimens.

Abbreviation: FBSA, flower-shaped bovine serum albumin. with the presence of $\mathrm{NaHCO}_{3}$. Energy-spectrum analysis of the specimen revealed that the element components in the FBSA with or without water wash changed dramatically (Figure 3): sodium was rich without wash while absent with wash (also means desalting), and the percentage of carbon was elevated from $16.10 \mathrm{wt} \%$ to $45.83 \mathrm{wt} \%$ with desalting. Carbon, nitrogen, and sulfur were detected in both specimens. We can conclude that the main component of flower-shaped particles was BSA. This also confirmed the existence of BSA in the shape of a flower.

We noted that there was precipitation of $\mathrm{Ca}_{2} \mathrm{CO}_{3}$ when $\mathrm{CaCl}_{2}$ was added to the solution. The emergent $\mathrm{Ca}_{2} \mathrm{CO}_{3}$ with the existence of $\mathrm{Na}_{2} \mathrm{CO}_{3} \cdot \mathrm{NaHCO}_{3} \cdot 2 \mathrm{H}_{2} \mathrm{O}$ (Figure 4 ) analyzed by X-ray diffraction might have enhanced the hardness of FBSA and changed it to a larger, thicker flower (Figure 4B,

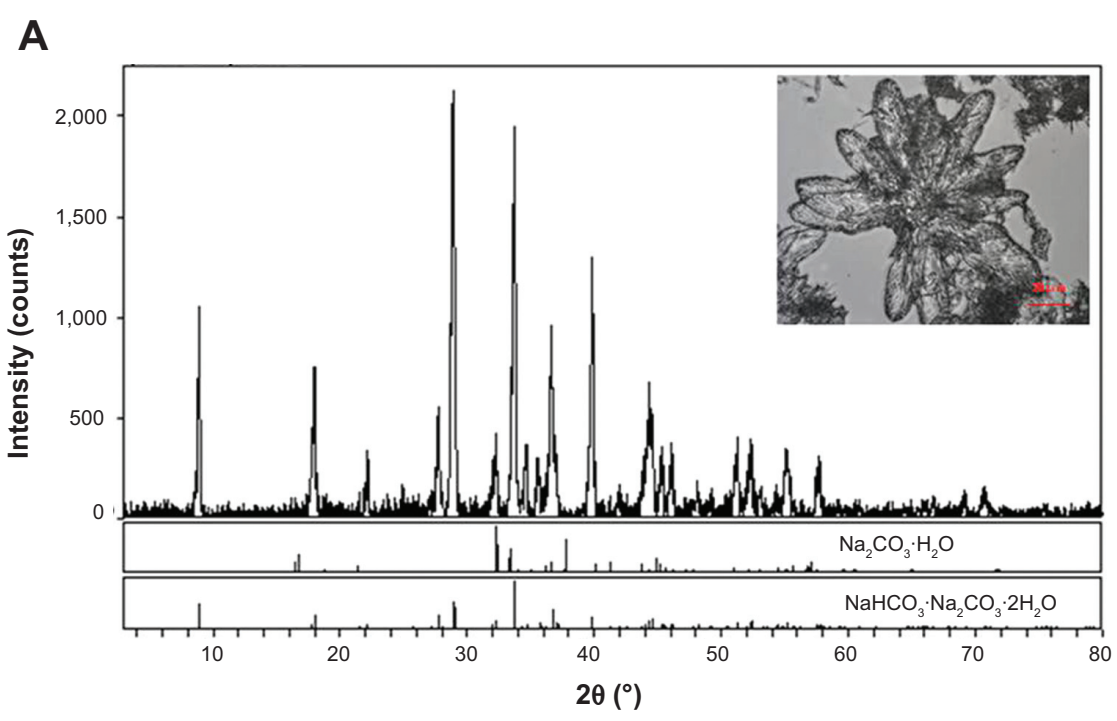

B

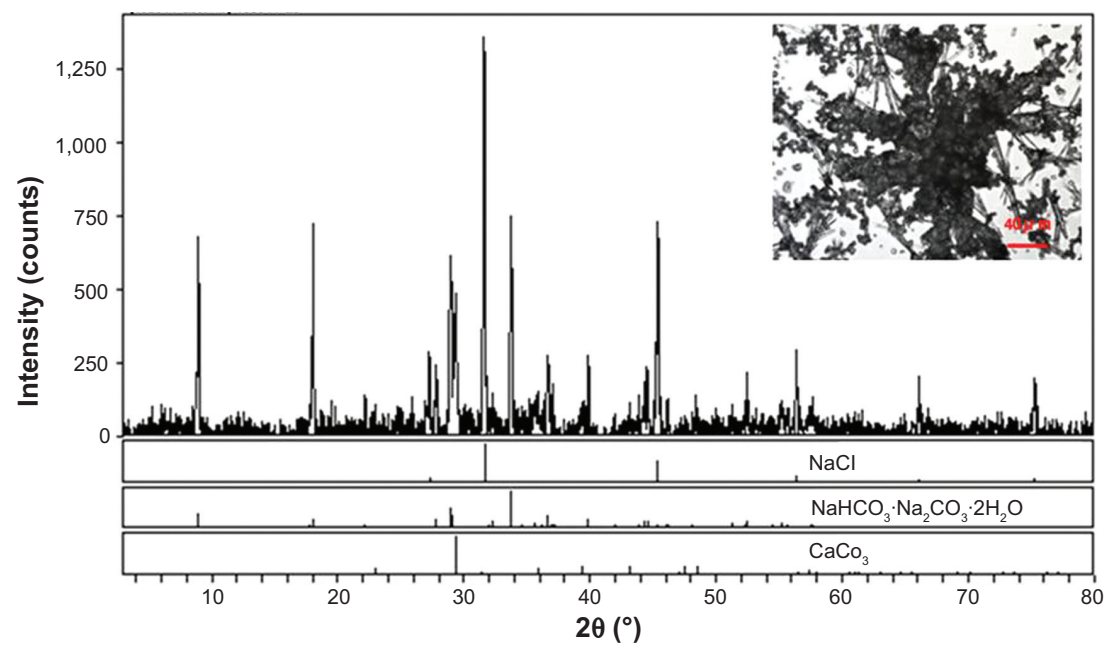

Figure 4 X-ray diffraction (XRD) analysis of FBSA.

Notes: The bright-field microscope images of FBSA (A [inset]) and FBSA with the addition of $\mathrm{CaCl}_{2}$ ( $\mathbf{B}$ [inset]) before fixation are presented. The XRD analysis demonstrates that the components of FBSA (A) are $\mathrm{Na}_{2} \mathrm{CO}_{3}$ and $\mathrm{Na}_{2} \mathrm{CO}_{3} \cdot \mathrm{NaHCO}_{3} \cdot 2 \mathrm{H}_{2} \mathrm{O}$. The components in FBSA (B) are $\mathrm{NaCl}_{2} \mathrm{Na}_{2} \mathrm{CO}_{3} \cdot \mathrm{NaHCO}_{3} \cdot 2 \mathrm{H}_{2} \mathrm{O}$, and $\mathrm{Ca}_{2} \mathrm{CO}_{3}$ when $\mathrm{CaCl}$ is added. Abbreviation: FBSA, flower-shaped bovine serum albumin. 
inset). The formation of $\mathrm{Ca}_{2} \mathrm{CO}_{3}$ may have been caused by the reaction between $\mathrm{CaCl}_{2}$ and $\mathrm{Na}_{2} \mathrm{CO}_{3} \cdot \mathrm{NaHCO}_{3} \cdot 2 \mathrm{H}_{2} \mathrm{O}$. On the other hand, the change in FBSA shape confirmed the template hypothesis. FBSA with enhanced hardness may have potential application in three-dimensional cell culture.

\section{Drug-loading and -release study}

We evaluated the drug-loading capacity and encapsulation efficiency of FBSA with the fluorescent dye RhB. The maximum drug-loading capacity of FBSA was $21.4 \%$ when $20 \mathrm{mg}$ of $\mathrm{RhB}$ was added. This was attributed to its high surface area and confinement of RhB on the flowers. The encapsulation efficiency was $41.3 \%$ at the most and then decreased slightly. It was likely due to the electrostatic interaction, hydrophobic interactions, or diffusion of model drug. ${ }^{10,23}$ According to Fick's law $(\mathrm{J}=-\mathrm{D} \mathrm{dc} / \mathrm{dx})$, higher loading capacity leads to a kinetic equilibrium state more quickly. The results in this report were consistent with the report of loading capacity of multidrug-loaded silk fibrillin described in Shi and Goh. ${ }^{10}$

The in vitro drug-release profiles of RhB from FBSA are shown in Figure 5. The profile showed that FBSA had a satisfactory controlled-release characteristic. The cumulative release of RhB over a period of 120 hours was $411.48 \mu \mathrm{g}$,

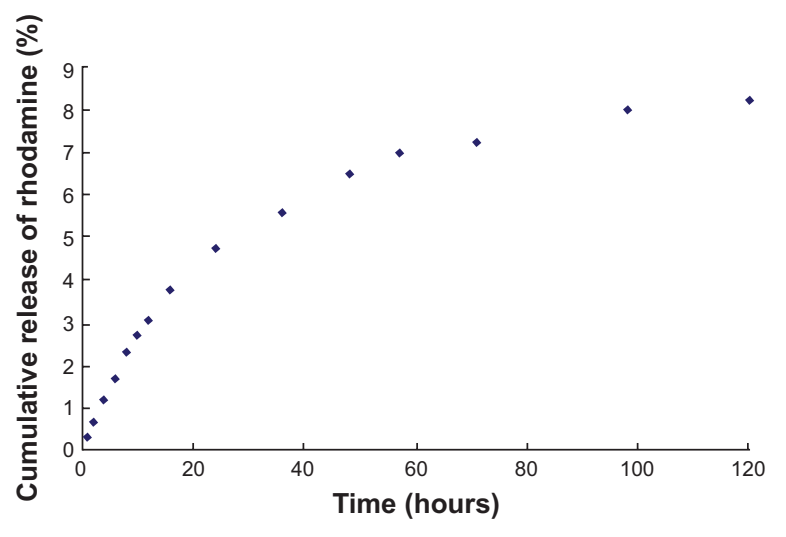

Figure 5 Controlled-release curve of rhodamine B from FBSA.

Abbreviation: FBSA, flower-shaped bovine serum albumin.

whereas the bounding amount of $\mathrm{RhB}$ was $5 \mathrm{mg}$, indicating good affinity between the FBSA and RhB. This was governed by Fickian diffusion, due to the electrostatic interaction between BSA and RhB, which restricted the release of positively charged $\mathrm{RhB}$ from negatively charged BSA in vitro. ${ }^{22}$

\section{In vitro cytocompatibility study}

To evaluate the cytocompatibility of FBSA, an in vitro experiment of FBSA-GA or FBSA-H against L929 cell lines was performed with CCK-8. Untreated cells (Figure 6E) served

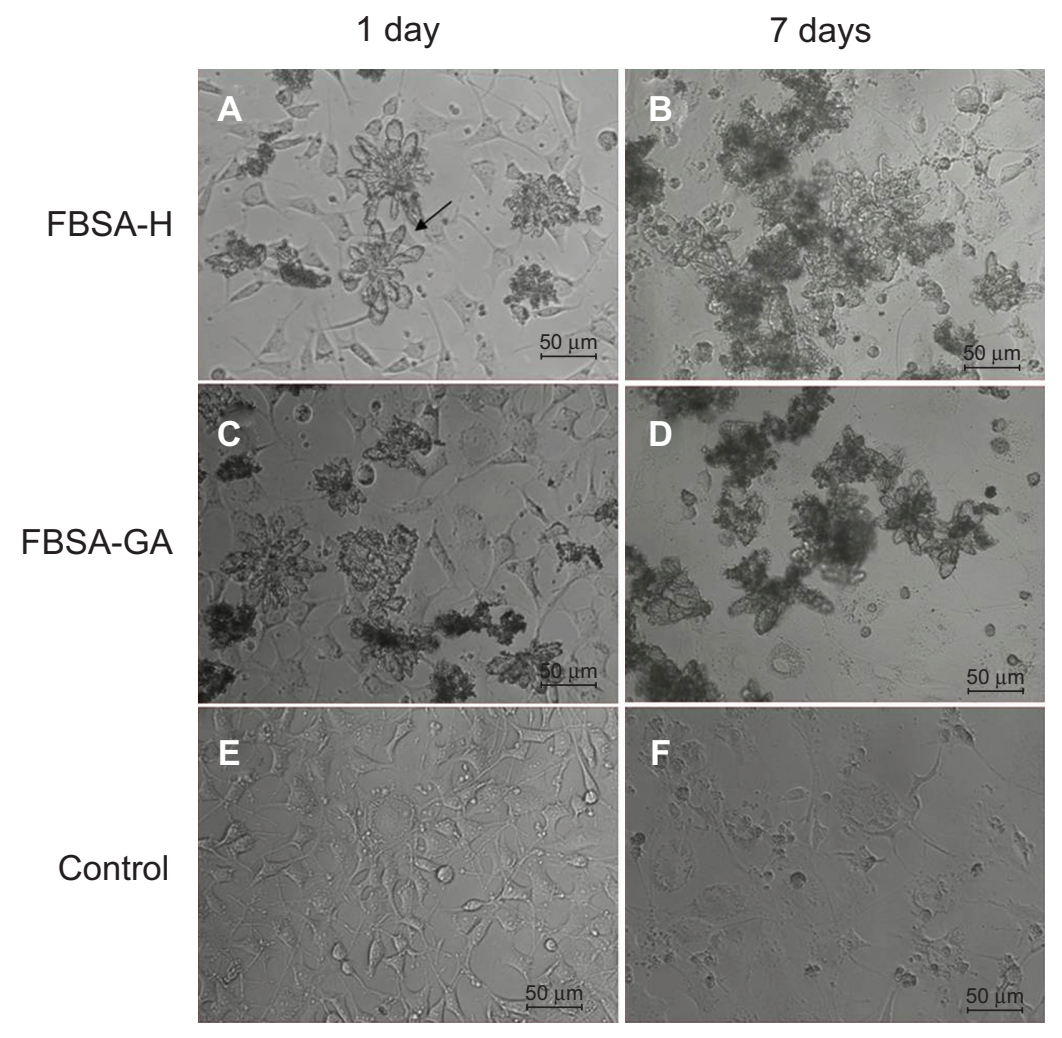

Figure 6 Morphology of L929 cells cultured with different conditions at different time points.

Notes: Cells were cultured with the addition of FBSA-H (A) and showed no change in cell proliferation (B). Seven days later, cells showed desirable viability. Cells cultured with the addition of FBSA-GA (C) showed obvious cell death (D). Cells cultured in normal conditions served as the control (E), and also showed cell death (F). Abbreviations: FBSA, flower-shaped bovine serum albumin; FBSA-H, heat-denatured FBSA; FBSA-GA, glutaraldehyde-fixed FBSA. 
as $100 \%$ cell viability. The relative viability of cells with the addition of FBSA-H (Figure 6A) and FBSA-GA (Figure 6C) was $101.0 \% \pm 5.97 \%$ and $94.5 \% \pm 12.8 \%$, respectively. These results indicated that FBSA-H had better cytocompatibility with no inherent cytotoxicity. The slight cytotoxicity of FBSA-GA was in agreement with that described in Speer et al. ${ }^{26} \mathrm{We}$ also found an individual cell passing through the petal of FBSA (Figure 6A, indicated by arrow). All of this suggested that FBSA might be a good scaffold for threedimensional cell culture, especially for tumor cells.

For further illustration of the biological activity of FBSA, we cultured the cells for 7 days without changing the medium. The addition of FBSA-H significantly prevented cells from death in the culture (Figure 6B). On the other hand, cell death could be observed obviously in the control (Figure 6F) and FBSA-GA (Figure 6D) groups. This indicated that the addition of FBSA-H might have the function of providing nutrition and promoting cell proliferation due to the hydrophobic domain of such natural protein, just like silk fibroin particles. ${ }^{10}$ FBSA-H had more advantages than other biomaterials, such as PLGA. However the nutrition properties of the proteins themselves on cell proliferation cannot compensate for the side effect of GA in the system, which explained the fact that most cells died in the FBSA-GA group (Figure 6D). This interesting finding shows that BSA is not only a soft material with good biocompatibility but also a nutrition provider. Further studies will focus on the assessment of FBSA drug delivery in the treatment of inner-ear disorders.

\section{Adhesive capability of FBSA on the RWM}

We isolated acoustic bullae with entire RWMs and compared the adhesive capability between FBSA and BSA NPs. SEM images revealed that FBSA adhered more firmly and deposited in multilayers (Figure 7B), while the BSA
NPs deposited in a monolayer (Figure 7A). This may be attributable to the larger adherent area of FBSA than BSA NPs. We assume that the higher surface area and adhesiveness make FBSA more suitable for local drug delivery.

\section{In vivo distribution of FBSA-RhB}

As for the good cytocompatibility and the higher activity of amino residue, ${ }^{24}$ FBSA-H particle-loaded $\mathrm{RhB}$ was chosen to investigate the penetration of RhB through the RWM. The soft surface made of natural protein had the advantage of constructing the carriers, because cells had the ability to sense the hardness of the culture surface. ${ }^{10,27,28}$

To confirm that the sustained release of RhB from FBSA was better than that from the BSA NPs, we injected FBSA$\mathrm{RhB}$ and BSA-NP-RhB into the right and left acoustic bullae of guinea pigs, respectively. Living images were taken at two time points: 0 and 48 hours. We observed that there were strong fluorescent signals in both acoustic bulla regions taken immediately after the operation. Two days (48 hours) later, there was still an obvious fluorescent signal in the right ear, while little in the left (Figure 8A and B). This implied that FBSA-RhB was retained longer in the acoustic bulla, and thus was more suitable for drug penetrating through the RWM along the concentration gradient in a controlledrelease profile.

The guinea pigs were then killed, and the RWMs separated for observation. We noted the biomaterials were still on the RWMs (Figure 8C), which indicated the continuous attachment of BSA. The fluorescent images revealed the obvious deposition of $\mathrm{RhB}$ (Figure $8 \mathrm{C}$, indicated by the arrow) with morphological change. We did not find flower-shaped particles on the RWM in SEM. There were two reasons we considered: one was the interference of salt crystals in phosphate-buffered saline, and the other was the
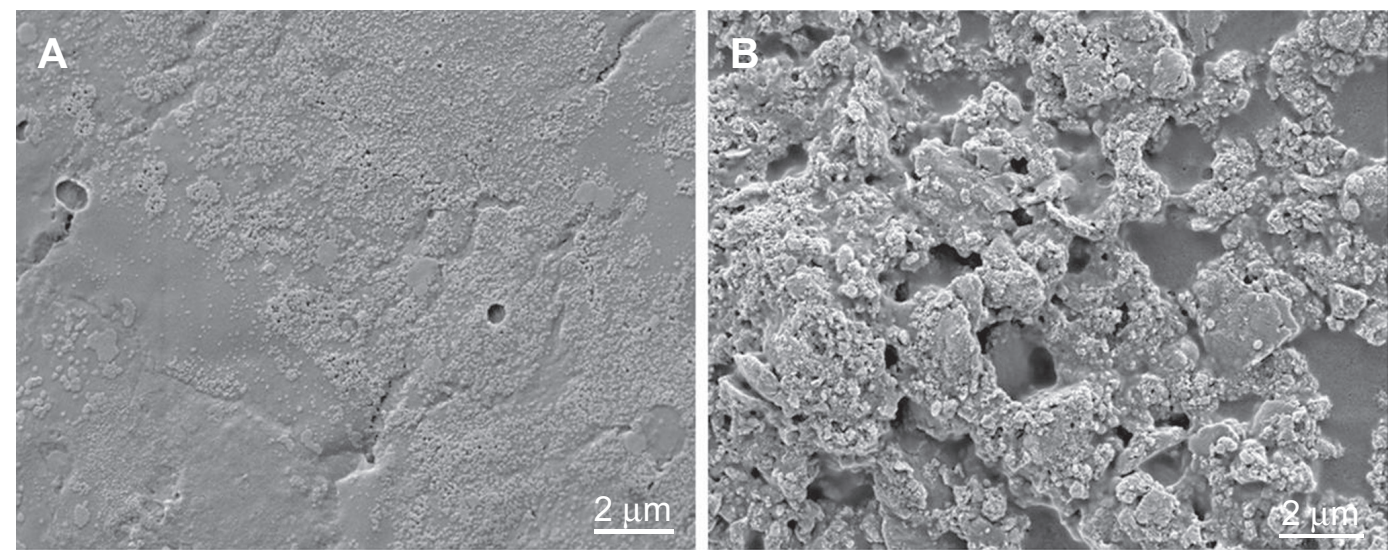

Figure 7 Evaluation of FBSA attachment on the RWM of guinea pigs.

Note: SEM images reveal the adherent difference between the BSA NPs (A) and FBSA (B).

Abbreviations: FBSA, flower-shaped bovine serum albumin; RWM, round-window membrane; NPs, nanoparticles; SEM, scanning electron microscopy. 

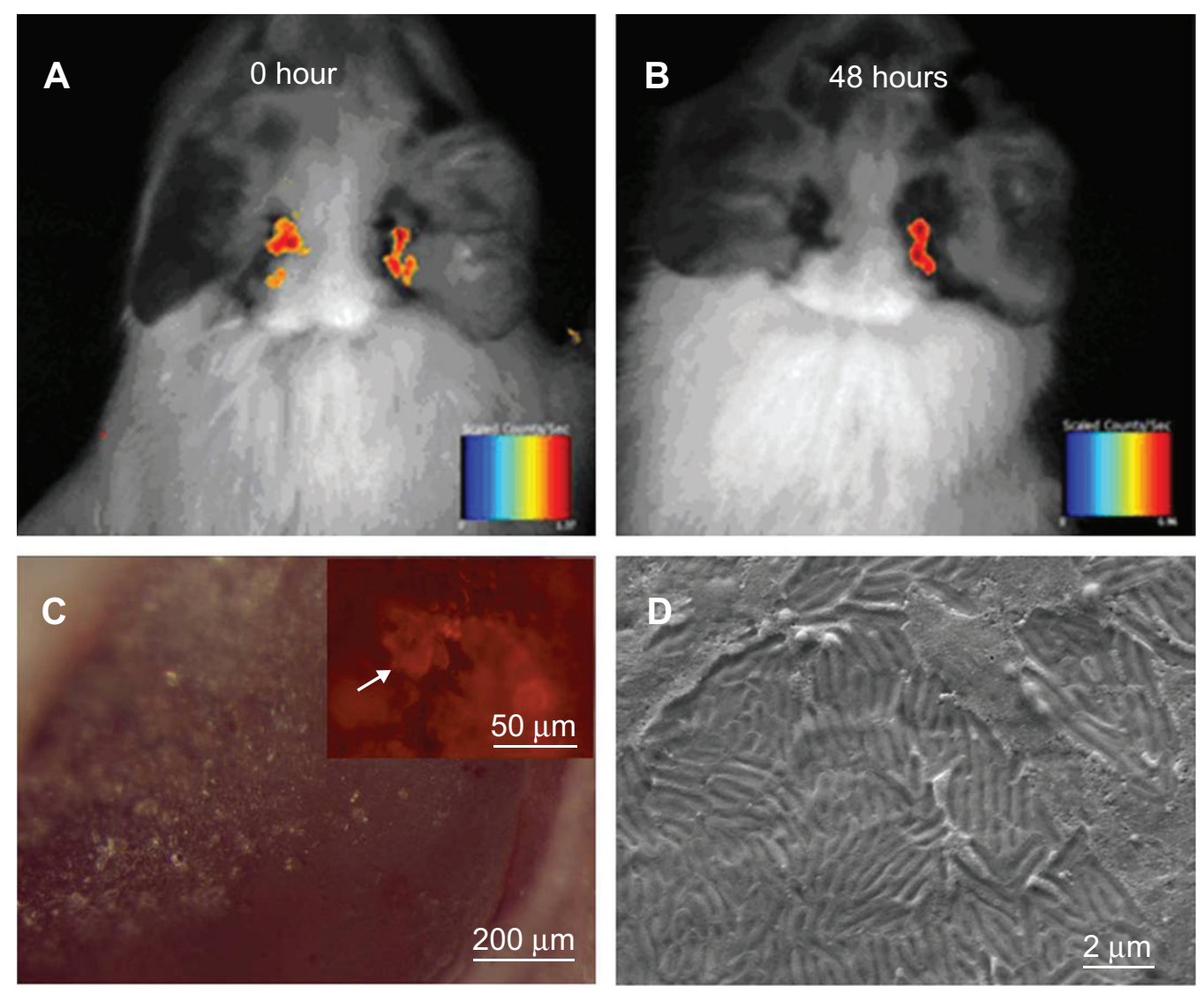

Figure 8 Images taken at different time points after FBSA-RhB was injected into the bullae of guinea pigs.

Notes: FBSA-RhB was retained in the bullae longer than the BSA-RhB NPs (A, B), viewed by living images. The living images were taken immediately (A) and 48 hours later (B). The RWM was observed by microscopes after living images. The biomaterials were still on the RWM, viewed by stereoscopic microscopy (C). The fluorescent image further revealed the obvious deposition of RhB (C [inset]). There was no FBSA on the RWM in the SEM image (D), taken several days later.

Abbreviations: FBSA, flower-shaped bovine serum albumin; FBSA-RhB, rhodamine B-loaded FBSA; RWM, round-window membrane; NPs, nanoparticles; SEM, scanning electron microscopy.

degradation of natural BSA by enzymes or other factors. This should be studied further.

\section{Conclusion}

In summary, FBSA microparticles were prepared by a modified desolvation method. We demonstrated that FBSA-H had great potential application for local drug delivery into the cochlea in the treatment of inner-ear diseases, due to its good biocompatibility, drug-loading capacity, controlled-release profile, and adhesive capacity. Further studies will focus on the evaluation of clinical drug-loaded FBSA, including prednisolone and dexamethasone sodium phosphate. We will evaluate their pharmacokinetics, pharmacodynamics, and delivery mechanism in a sensorineural hearing-loss model. FBSA also shed light on local drug delivery in the treatment of such inner-ear disorders as tinnitus, sensorineural hearing loss, and Ménière's disease.

\section{Acknowledgments}

This work was supported by the Project in the Eleventh Five-Year Plan of the Second Artillery General
Hospital, the National Key Scientifc Program of China (2006CB933300), the Peking Union Medical College Youth Fund (3332013109, 2012D08), and the Fundamental Research Funds for the Central Universities.

\section{Disclosure}

The authors report no conflicts of interest in this work.

\section{References}

1. Lajud SA, Han Z, Chi FL. A regulated delivery system for inner ear drug application. J Control Release. 2013;166(3):268-276.

2. Schuknecht HF. Ablation therapy for the relief of Ménière's disease. Laryngoscope. 1956;66(7):859-870.

3. Kopke RD, Hoffer ME, Wester D, O'Leary MJ, Jackson RL. Targeted topical steroid therapy in sudden sensorineural hearing loss. Otol Neurotol. 2001;22(4):475-479.

4. Balough BJ, Hoffer ME, Wester D, O’Leary MJ, Brooker CR, Goto M. Kinetics of gentamicin uptake in the inner ear of Chinchilla langier [sic] after middle-ear administration in a sustained-release vehicle. Otolaryngol Head Neck Surg. 1998;119(5):427-431.

5. Horie RT, Sakamoto T, Nakagawa T, et al. Sustained delivery of lidocaine into the cochlea using polylactic/glycolic acid microparticles. Laryngoscope. 2010;120(2):377-383.

6. Ge J, Lei JD, Zare RN. Protein-inorganic hybrid nanoflowers. Nat Nanotechnol. 2012;7(7):428-432. 
7. Tan J, Wang YJ, Yip XP. Nanoporous peptide particles for encapsulating and releasing neurotrophic factors in an animal model of neurodegeneration. Adv Mater. 2012;24(25):3362-3366.

8. Li RF, Li XM, Liu LR, Zhou ZM, Tang HB, Zhang QQ. High-yield fabrication of PLGA non-spherical microarchitectures by emulsionsolvent evaporation method. Macromol Rapid Commun. 2010; 31(22): 1981-1986.

9. Mitragotri S, Lahann J. Physical approaches to biomaterial design. Nat Mater. 2009;8(1):15-23.

10. Shi PJ, Goh JC. Release and cellular acceptance of multiple drugs loaded silk fibroin particles. Int J Pharm. 2011;420(2):282-289.

11. Xu Y, Palchoudhury S, Qin Y, Macher T, Bao Y. Make conjugation simple: a facile approach to integrated nanostructures. Langmuir. 2012;28(23):8767-8772.

12. Zhou ZM, Anselmo AC, Mitragotri S. Synthesis of protein-based, rodshaped particles from spherical templates using layer-by-layer assembly. Adv Mater. 2013;25(19):2723-2727.

13. Geng Y, Dalhaimer P, Cai SS. Shape effects of filaments versus spherical particles in flow and drug delivery. Nat Nanotechnol. 2007;2(4): 249-255.

14. Yoo JW, Doshi N, Mitragotri S. Endocytosis and intracellular distribution of PLGA particles in endothelial cells: effect of particle geometry. Macromol Rapid Commun. 2010;31(2):142-148.

15. Haghgooie R, Toner M, Doyle PS. Squishy non-spherical hydrogel microparticles. Macromol Rapid Commun. 2010;31(2):128-134.

16. Acharya G, Shin CS, McDermott M, et al. The hydrogel template method for fabrication of homogeneous nano/microparticles. J Control Release. 2010;141(3):314-319.

17. Gratton SE, Napier ME, Ropp PA, Tian S, DeSimone JM. Microfabricated particles for engineered drug therapies: elucidation into the mechanisms of cellular internalization of PRINT particles. Pharm Res. 2008;25(12):2845-2852.
18. Cui HG, Chen ZY, Zhong S, Wooley KL, Pochan DJ. Block copolymer assembly via kinetic control. Science. 2007;317(5838):647-650.

19. Rodrigues NF, van Tilburg Bernardes E, Rocha RP. Bovine serum albumin nanoparticle vaccine reduces lung pathology induced by live Pseudomonas aeruginosa infection in mice. Vaccine. 2013;31(44): 5062-5066.

20. Elzoghby AO, Samy WM, Elgindy NA. Albumin-based nanoparticles as potential controlled release drug delivery systems. J Control Release. 2012;157(2):168-182.

21. Elsadek B, Kratz F. Impact of albumin on drug delivery - new applications on the horizon. J Control Release. 2012;157(1):4-28.

22. Zhang X, Chen G, Wen L, et al. Novel multiple agents loaded PLGA nanoparticles for brain delivery via inner ear administration: in vitro and in vivo evaluation. Eur J Pharm Sci. 2013;48(4-5):595-603.

23. Lammel AS, Hu X, Park SH, Kaplan DL, Scheibel TR. Controlling silk fibroin particle features for drug delivery. Biomaterials. 2010;31(16): 4583-4591.

24. Liu M, Zhou ZM, Wang XF, et al. Formation of poly(L,D-lactide) spheres with controlled size by direct dialysis. Polymer. 2007;48(19): 5767-5779.

25. Zhou ZM, Xu J, Liu XQ, et al. Non-spherical racemic polylactide microarchitectures formation via solvent evaporation method. Polymer. 2009;50(15):3841-3850.

26. Speer DP, Chvapil M, Eskelson CD, Ulreich J. Biological effects of residual glutaraldehyde in glutaraldehyde-tanned collagen biomaterials. J Biomed Mater Res. 1980;14(6):753-764.

27. Discher DE, Janmey P, Wang YL. Tissue cells feel and respond to the stiffness of their substrate. Science. 2005;310(5751):1139-1143.

28. Doshi N, Zahr AS, Bhaskar S, Lahann J, Mitragotri S. Red blood cellmimicking synthetic biomaterial particles. Proc Natl Acad Sci US A 2009;106(51):21495-21499.
International Journal of Nanomedicine

\section{Publish your work in this journal}

The International Journal of Nanomedicine is an international, peerreviewed journal focusing on the application of nanotechnology in diagnostics, therapeutics, and drug delivery systems throughout the biomedical field. This journal is indexed on PubMed Central, MedLine, CAS, SciSearch $\AA$, Current Contents $₫ /$ Clinical Medicine,

\section{Dovepress}

Journal Citation Reports/Science Edition, EMBase, Scopus and the Elsevier Bibliographic databases. The manuscript management system is completely online and includes a very quick and fair peer-review system, which is all easy to use. Visit http://www.dovepress.com/ testimonials.php to read real quotes from published authors. 